
\title{
R Reserach S Suare \\ Efficacy of Guizhi Fuling Wan for Primary dysmenorrhea: protocol for a randomised controlled trial
}

\section{Du Yun}

China Three Gorges University https://orcid.org/0000-0002-1723-0473

\section{Yatong Li}

China Three Gorges University

\section{Xianyun Fu}

China Three Gorges University

Yanan Luo ( $\sim$ luoyanan@ctgu.edu.cn )

China Three Gorges University https://orcid.org/0000-0001-8345-6270

\section{Chenjie Li}

The Second People's Hospital of Yichang

\section{Study protocol}

Keywords: Guizhi Fuling Wan, Primary dysmenorrhea, Randomized controlled trial, Placebo control

Posted Date: April 27th, 2021

DOI: https://doi.org/10.21203/rs.3.rs-462906/v1

License: (c) (1) This work is licensed under a Creative Commons Attribution 4.0 International License. Read Full License 


\section{Abstract}

\section{Background}

Primary dysmenorrhea (PD) is one of main gynecological complaints in women of child-bearing age, but there are limited effective treatments available. Guizhi Fuling Wan (GFW), one of the most widely known traditional Chinese medicine (TCM) herbal formulas, has been the treatment of choice for gynecological disease in China. A growing number of studies have shown that GFW is beneficial for patients with PD. However, the quality of evidence is limited, and there are few studies on the treatment of PD by GFW with specific TCM syndromes. Therefor we will conduct a randomized controlled trial to explore the efficacy and safety of GFW for PD patients with heat-burning blood-stasis syndrome.

Methods and analysis

This study is designed as a randomized, double-blinded, placebo-controlled clinical trial. Eligible patients will be randomly assigned to the GFW group (patients receive GFW) and the control group (patients receive a matching placebo) in a 1:1 ratio. The participants will receive either GFW or placebo, twice daily for 3 menstrual cycles with a 3-month follow-up. The primary outcome is the pain intensity reduction measured by a Visual Analog Scale. The secondary outcomes is the Cox Menstrual Symptom Scale, the Self-rating Depression Scale, the Self-rating Anxiety Scale, the 12-item Short-Form Health Survey and the dysmenorrhea diarymenstrual pain.. All outcomes are assessed at baseline, and the 4th, 8th, and 12th week during the interventions, as well as at the 16th, 20th and 24th week follow-up. Any adverse events will be recorded throughout the study.

\section{Discussion}

This is the first double-blinded RCT to assess the effectiveness and safety of GFW in treating PD with the heat-burning blood-stasis syndrome. The scientific and rigorous methodology design of this trial will provide significant evidence regarding the use of GFW to treat PD in future.

Trial registration

Chinese Clinical Trial Registry, ID: ChiCTR2000034118. Regisrered on 25 June 2020.

\section{Background}

Primary dysmenorrhea (PD) is a common gynecological disorder among reproductive women, with pain caused by menstruation without pelvic pathology as the main symptom. It has been reported that almost $40-75 \%$ of women may suffer from menstrual cramps accompanied by some associated symptoms, including weakness, diarrhea, nausea, and headache [2-5]. Meanwhile, women with PD are more likely to experience irritability, reducing their quality of life [5]. Over time, severe PD even leads to socioeconomic problems considerably, with $1 / 3$ to $1 / 2$ of patients missing school or work at least once per cycle [6]. 
Although there are numerous studies about PD, the pathomechanism of dysmenorrhea is not fully understood. Previous research indicates that prostaglandins (PGs) play a significant role in dysmenorrhea's pathomechanism, and there are complex biochemical reactions between the proinflammatory factors, endocrine, and vascular [7]. At present, the most frequently prescribed treatments are nonsteroidal anti-inflammatory drugs (NSAIDs) and oral contraceptives [8-9]. Despite their well-recognized efficacy, professional societies recommend using NSAIDs with caution, balancing the various potential gastrointestinal, cardiovascular, and renal adverse events [10]. Oral contraceptives are also not a widely accepted treatment due to the risk of venous thrombosis and hormone-dependent diseases [11]. Given this situation, it is necessary to seek alternative therapies to meet clinical needs.

Chinese herbal medicine (CHM) is considered as a well-accepted alternative therapy for PD in China. Guizhi Fuling Wan (GFW) is one of the most widely known CHM formulas composed of five herbs, including Cinnamon Twig, Poria cocos, Cortex Moutan, Radix Paeoniae Rubra, and Peach kernel. It is widely used to treat dysmenorrhea, endometriosis, uterine fibroids, pelvic inflammation, mammary gland hyperplasia, and other gynecological diseases for almost two thousand years since the Eastern Han Dynasty (third century A.D.) [13]. In recent studies, GFW's pharmaceutical ingredient has been shown to affect suppressing uterus contraction, prostaglandin level reduction, and correction of luteal insufficiency, demonstrating the potential of promising drugs for PD [14-16]. It also suggests that the GFW had therapeutic effects on PD rats via the regulation of multiple metabolic pathways [17]. GFW has also shown promising results and no severe adverse reactions were found in some randomized trials. At present, the relevant studies are prospective long-term clinical observational studies, while there are few RCTs [18]. Previous RCT research designs always followed the 2010 version of CONSORT standard, and rarely use the latest CONSORT extension for Chinese herbal medicine formulas 2017, which can better ensure the quality and authenticity of TCM research [20]. In addition, most trials did not diagnose patients with TCM dialectical classification, which is the core of TCM treatment practice [19]. Therefore, a randomized controlled trial (RCT) targets specific TCM pattern and is needed to assess the benefit of GFW on PD patients.

Thus, we have designed a randomized, double-blinded, placebo-controlled trial to evaluate the efficacy and safety of GFW for PD patients with heat-burning blood-stasis syndrome.

\section{Methods}

\section{Design and setting}

This study is a single-centered, randomized, double-blind, and placebo-controlled trial to evaluate GFW's efficacy and safety for PD. The survey designed will comply with the Declaration of Helsinki (2013 version) and Consolidated Standards of Reporting Trials guidelines Extension for Chinese Herbal Medicine Formulas 2017 (CONSORT CHM Formula) [1]. Figure 1 provides the flow diagram. This study's protocol is prepared based on the Standardized Protocol Items: Recommendations for Interventional Trials (SPIRIT) guidelines [21]. The Ethical Committee of The Second People's Hospital of Yichang has 
approved the protocol (Approval No.2020001), which was registered on the Chinese Clinical Trials Registry in 2020 (Registration No. ChiCTR2000034118.).

The trial will be conducted at the Second People's Hospital of Yichang. Participants will be required to complete a screening test, and eligible patients will enter a baseline period of one week, during which they will be asked to complete a dysmenorrhea diary and record-related symptom scores. Subjects meeting the including criteria will be randomly allocated to either the GFW group or the placebo control group. Participants in both groups will receive treatment for three menstrual cycles and three months of followup through telephone calls or social media. Patient enrollment will start in late October 2020 and is expected to end in December 2021 (Flowchart of the study design see Fig. 1 and the assessment schedule see table.1).

\begin{tabular}{|c|c|c|c|c|c|c|c|c|}
\hline \multirow[b]{3}{*}{ Time point (menstrual cycle) } & \multicolumn{8}{|c|}{ Study period } \\
\hline & \multirow{2}{*}{$\begin{array}{r}\text { Enrollment } \\
-1 \\
\end{array}$} & \multirow{2}{*}{$\begin{array}{r}\text { Allocation } \\
0 \\
\end{array}$} & \multicolumn{3}{|c|}{$\begin{array}{c}\text { Treatment } \\
\text { period }\end{array}$} & \multicolumn{3}{|c|}{$\begin{array}{l}\text { Follow-up } \\
\text { period }\end{array}$} \\
\hline & & & 1 & 2 & 3 & 4 & 5 & 6 \\
\hline \multicolumn{9}{|l|}{ Enrollment: } \\
\hline \multirow{5}{*}{$\begin{array}{l}\text { Eligibility screen } \\
\text { Informed consent } \\
\text { Physical examination } \\
\text { medical history } \\
\text { Allocation }\end{array}$} & $\times$ & & & & & & & \\
\hline & & $\times$ & & & & & & \\
\hline & $\times$ & & & & & & & \\
\hline & $\times$ & & & & & & & \\
\hline & & $\times$ & & & & & & \\
\hline \multicolumn{9}{|l|}{ Interventions: } \\
\hline \multicolumn{9}{|l|}{ GFW group } \\
\hline Placebo control group & & & •- & & $\longrightarrow$ & & & \\
\hline Assessments: & & & & & & & & \\
\hline \multirow{2}{*}{$\begin{array}{l}\text { Menstrual pain intensity } \\
\text { CMSS }\end{array}$} & $\times$ & & $\times$ & $x$ & $\times$ & $x$ & $x$ & $\times$ \\
\hline & $\times$ & & $\times$ & $\times$ & $\times$ & $\times$ & $\times$ & $\times$ \\
\hline SDS & $x$ & & $\times$ & $\times$ & $\times$ & $x$ & $x$ & $\times$ \\
\hline \multirow{2}{*}{$\begin{array}{l}\text { SAS } \\
\text { SF-12 }\end{array}$} & $\times$ & & $\times$ & $\times$ & $\times$ & $\times$ & $\times$ & $\times$ \\
\hline & $\times$ & & $\times$ & $\times$ & $\times$ & $x$ & $\times$ & $\times$ \\
\hline \multirow{2}{*}{$\begin{array}{l}\text { Dysmenorrhea diary } \\
\text { AEs }\end{array}$} & $\times$ & & $\times$ & $\times$ & $\times$ & $\times$ & $\times$ & $\times$ \\
\hline & & & $\times$ & $x$ & $\times$ & $x$ & $x$ & $x$ \\
\hline SAE & & & $\times$ & $\times$ & $\times$ & $x$ & $\times$ & $\times$ \\
\hline
\end{tabular}

CMSS Cox Menstrual Scale, SDS the Self-rating Depression Scale, $S A S$ the Self-rating Anxiety Scale, SF-12 the 12-item Short-Form Health Survey, $A E s$ adverse events, SAE Serious Adverse Events.

\section{Participants}




\section{Inclusion criteria}

Participants meeting the following criteria will be included: (1) nulliparous women of 18 to 30 years old; (2) meet the diagnostic criteria of PD by Chinese Obstetrics And Gynecology(3rd edition); (3) accord with the TCM diagnosis of "heat-burning blood-stasis syndrome"; (4) pain symptoms last from 6 months to 15 years; (5) a VAS score of menstrual pain $\geq 40 \mathrm{~mm}$ at least for three consecutive menstrual cycles; (6) signature of an informed consent form.

\section{Exclusion criteria}

Participants would be excluded if they meet any of the following criteria: (1) unable to complete or comply with the study; (2) imaging examination suspects secondary dysmenorrhea; (3) pregnant during the period of the trial; (4) unable to cooperate with the questionnaire due to intellectual or mental disorder; (5) history or presence of clinically relevant severe cardiovascular and cerebrovascular diseases, liver and kidney dysfunction, mental illness, or life-threatening conditions; (6) treatment with analgesics, antidepressants, or psychotropic drugs for PD in the past month; (7) are participating in the other clinical trials of drugs.

Potentially eligible participants will be screened by the research staff through physical examination and clinical tests. All participants will be required to sign an informed consent form before enrollment. Patients' information will be kept confidential, and the patient has the right to withdraw it at any time without prejudice.

\section{Interventions}

Participants in the GFW group receive GFW (herb granule, 30gper packet) treatment. It has the effect of clearing heat and dispelling dampness, blood circulation and removing blood stasis and clearing away heat and blood. It consists of five natural herbs: Guizhi (Cinnamon Twig) 6g, Fuling (Poria Cocos) 6g, Mudanpi (Cortex Moutan) 6g, Chishao (Radix Paeoniae Rubra) 6g, Taoren (Peach Kernel) 6g [22].

Participants in the control group receive GFW simulated preparation composed of Maiya (Hordei Fructus Germinatus), edible and medicinal. The dosage form, color, appearance, and packaging of the GFW fake preparation are consistent with GFW in the treatment group [23].

Both GFW formula and GFW simulated preparation are granules provided by Beijing Tcmages Pharmaceutical Co. Ltd. (Beijing, China) and distributed by the Second People's Hospital of Yichang. The production process will be subject to quality control to ensure that the packaging has consistent purity, microbial content, weight, and other physical characteristics.

Patients in the two groups will be given oral medication every 12 hours a day, $30 \mathrm{~g}$ each time, from 7 days before menstruation to 3 days after menstruation for three menstrual cycles. Participants were allowed to use NSAIDs such as ibuprofen if required, and the outcome assessment is not scheduled in the next 48 hours [24]. The use of all drugs should be documented in the case report form (CRF). Medicine will be 
kept sealed in a cool, dry, and secure locked place, and patients will be asked to record menstrual pain and acute medication use in a dysmenorrhea diary throughout the trial. Besides, if patients have intolerable symptoms, the attending doctor will treat the patient according to the actual situation and estimate whether adverse events are related to the study drugs; if so, they will decide to terminate the experiment.

\section{Outcomes and follow-up}

Primary outcome

The primary outcome will be menstrual pain intensity measured by the visual analog scale (VAS) scores. Participants will be asked to indicate a perception of pain intensity (most commonly) scored from 10 to 100 (0, no pain; 100 , maximum) [25].

Secondary outcomes

The secondary outcomes include the Cox Menstrual Symptom Scale (CMSS), the Self-rating Depression Scale (SDS), the Self-rating Anxiety Scale (SAS), the 12-item Short-Form health survey (SF-12), and the dysmenorrhea diarymenstrual pain.

Safety assessments

Safety is assessed by adverse events and serious adverse events.

The primary and secondary outcomes will be recorded at baseline, treatment period and the follow-up periods. Safety assessments will be detected at the treatment period and the follow-up periods.

The assessments will be performed in a separate space in the outpatient department. All of the outcome assessors will be trained in conducting interviews and performing measurements before the study. The following measures will be taken to optimize participants' retention: free examination and treatment will be provided during the survey; appointments will be arranged flexibly to suit the patients' schedule [26].

The participants will be contacted monthly by telephone or social media to monitor their intake of the granules. If someone has taken less than $80 \%$ of the medicine, they would be considered non-compliant [27].

\section{Randomization and allocation concealment}

Eligible participants will be randomly assigned to either the treatment group or the control group at a 1:1 ratio after the screening visit [22]. Random numbers will be generated by a researcher who is not practicing throughout the study, using the SPSS Statistics for Windowa, Version 22.0 (SPSSInc., Chicago, III., USA). For allocation concealment, the name of each group (A or B), prepared by another researcher (not involved in the study), will be written on a card and placed in opaque envelopes labeled with sequential numbers, according to the sample size. Then the envelopes are sequentially opened for every 
new participant to determine the group. The envelopes will be placed numerically in a safe place until the study is completed.

\section{Blinding}

In this double-blind trial, none of the participants, clinical practitioners, the outcome evaluators, the data manager, and statistician will be aware of group assignments until the end of the study period. The placebo granule will be matched to the GFW herb granule in color, taste, smell, and outer packaging. Elimination of blindness will only be considered in cases of serious medical emergencies.

\section{Sample size}

Assuming a difference of $1 \mathrm{~cm}$ on the VAS as the minimum clinically relevant difference and SDs was 1.67 , consistent with those observed in previous similar studies overall 2-tailed 0.05 level significance $90 \%$ power [29-30]. The ratio between the two groups at 1:1, a minimum sample size of 116 patients (58 patients in each group) is needed. A minimum sample size of 128 patients (64 patients per arm) at the baseline will be required to allow for a maximum dropout tolerance of $10 \%$.

\section{Data collection and management}

Data will be collected from the dysmenorrhea daily of symptoms recorded by participants, the CRF tables, and TCM syndrome scores recorded by the trial team [31]. All patients will be invited to answer the data administrator's self-administered electronic questionnaire [32]. All filled questionnaires will be checked for completeness by data collectors, and questionnaires with missing items will be returned to the patient in time to maximize the richness of the information and minimize recall bias [32, 33]. Regarding the recruitment rate, the study screening log will record the reasons for unqualified and non-recruitment patients. Retention and reasons for the withdrawal of participants throughout the trial will be documented [34]. The outcome measures will be described according to Fig. 1.

Qualified sites, and well-trained assessors are crucial factors that ensure the quality of a clinical trial [35, 36]. An independent data monitoring committee will be composed of two expert clinicians with experience in conducting and monitoring clinical trials [37] and they will check the data routinely. Any incomplete data will be coded as unknown, missing, or not applicable. Confidentiality of patient data will be maintained throughout the study. Trial data will be accessible only to the investigator team [38].

\section{Data analysis}

At the end of all measurements' data collection, statistical analyses will be performed by SPSS Statistics for Windowa, Version 22.0 (SPSSInc., Chicago, III., USA) [39]. we will descriptively summarise patient characteristics, outcome variables and the adverse events. Continuous variables, such as age and disease course, will be presented as mean \pm standard deviation (SD) and analysed by independentsample T test. Quantitative variables such as baseline variables, sociodemographic data will be analyzed using $\chi^{2}$ tests or Mann-Whitney $U$ tests. The repeated measurement variables, such as the primary 
outcome and second outcome, are analyzed by Repeated ANOVA or generalized estimation equations (GEE).

Both the full analysis set and per-protocol analysis set will be used simultaneously to evaluate the effect and safety of GFW for PD. The last-observation-carried-forward method will be used for missing data [40]. Statistical significance will be set at $P<0.05$.

\section{Discussion}

GFW, the classical CHM formula, is regarded as a promising therapeutic Chinese herb for the treatment of PD by regulating pros-taglandins (PGs) oversecretion [14-16], reducing hyperalgesia, myoconstriction, vasoconstriction, inflammatory pain and uterine ischemia. However, the current RCT that support the efficacy of GFW in the treatment of PD still has some limitations, such as the absence of syndrome differentiation for patient and small sample size [18]. As a result, the efficacy of GFW on patients with PD needs to be verified by higher quality RCT. Therefore, we intend to verify the effectiveness and safety of GFW in the treatment of PD with the heat-burning blood-stasis syndrome through a double-blinded RCT.

Treatment based on syndrome differentiation has always been the core of TCM clinical diagnosis and treatment. Accurate syndrome differentiation and treatment can not only guide the medication, but also improve the curative effect. At present, researchers pay more and more attention to the treatment based on syndrome differentiation, and gradually bring it into clinical trials [19]. Through long-term clinical observation, we found that GFW is effective in the treatment of dysmenorrhea, especially in the patients with "heat burning blood stasis syndrome". Therefore, in order to follow the basic principles of TCM treatment, this study included the PD with the heat-burning blood-stasis syndrome. In order to ensure the reliability of TCM dialectical classification, two experienced doctors will provide TCM diagnosis for patients.

Appropriate placebo can reduce implementation bias. In our study, the placebo is GFW mimetic preparation, which is matched with GFW granules in terms of texture, dosage and appearance, as well as outer packaging. Those supervising or administering outcome assessors will be blinded to the group allocation. Also, standardized and validated questionnaires will be distributed to ensure credible measurement of patient health status.

Our study also has a few limitations. First, in order to improve patient compliance and facilitate patient recruitment, we enrolled patients from China Three Gorges University, which would lead to selective bias to some extent. However, the university is a comprehensive university with students from all over the country. Second, the placebo and GFW were identical in texture, dosage and appearance, but there are slight differences in smell and taste between them, which may have some implementation bias.

In sum, this trial is the first study designed to demonstrate the efficacy and safety of GFW in treating PD patients with heat-burning blood-stasis syndrome, compared with a placebo. The scientific and rigorous methodologic design of this trial is expected to provide reliable evidence for the efficacy and safety of the GFW in treating PD, and provide reference for clinical practice. 


\section{Declarations}

\section{Trial status}

Participant recruitment is currently ongoing.

\section{Acknowledgements}

The authors thank all of the collaborators and participants of the study.

\section{Funding}

This trial was supported in part by the National Natural Science Foundation of China (grant number 81973897).

\section{Availability of data and materials}

All data and material supporting our findings can be obtained from the main author.

\section{Authors' contributions}

F X Y and L Y N supervised the study and obtained study funding and ethical approval. F XY, L Y N and $L$ $\mathrm{C} \mathrm{J}$ conceived and designed the study and are responsible for the coordination of the study. D Y and $\mathrm{F} \mathrm{X}$ Y drafted and edited the manuscript. L Y T and L C J participated in the work of enrolling the patients and collecting the data. All authors have read and approved the final manuscript.

\section{Ethics and dissemination}

The protocol has been approved by the Ethical Committee of The Second People's Hospital of Yichang. Any modifications to the research protocol will be notified to this Human Research Ethics Committee. Informed consent will be obtained from each subject prior to enrollment. This trial conforms to the Declaration of Helsinki (2013 version) and Consolidated Standards of Reporting Trials guidelines Extension for Chinese Herbal Medicine Formulas 2017 (CONSORT CHM Formula)(1)[1].

\section{Consent for publication}

No personal data will be published, and the informed consent form will include permission to publish group data. Results will be disseminated in peer-reviewed publications and published in international journals.

\section{Competing interests}

The authors declare that they have no competing interests.

\section{References}


1. Cheng CW. CONSORT Extension for Chinese Herbal Medicine Formulas 2017: Recommendations, Explanation, and Elaboration. ANN INTERN MED. 2017;167(2):112-21.

2. Fernandez-Martinez E, Onieva-Zafra MD, Parra-Fernandez ML. Lifestyle and prevalence of dysmenorrhea among Spanish female university students. PLOS ONE. 2018;13(8):e201894.

3. Hu Z, Tang L, Chen L, Kaminga AC, Xu H. Prevalence and Risk Factors Associated with Primary Dysmenorrhea among Chinese Female University Students: A Cross-sectional Study. J Pediatr Adolesc Gynecol. 2020;33(1):15-22.

4. Zurawiecka M, Wronka I. Association of primary dysmenorrhea with anthropometrical and socioeconomic factors in Polish university students. J Obstet Gynaecol Res. 2018;44(7):1259-67.

5. Chen L, Tang L, Guo S, Kaminga AC, Xu H. Primary dysmenorrhea and self-care strategies among Chinese college girls: a cross-sectional study. BMJ OPEN. 2019;9(9):e26813.

6. Al-Matouq S, Al-Mutairi H, Al-Mutairi O, Abdulaziz F, Al-Basri D, Al-Enzi M, et al. Dysmenorrhea among high-school students and its associated factors in Kuwait. BMC PEDIATR. 2019;19(1):80.

7. Barcikowska Z, Rajkowska-Labon E, Grzybowska ME, Hansdorfer-Korzon R, Zorena K. Inflammatory Markers in Dysmenorrhea and Therapeutic Options. INT J ENV RES PUB HE. 2020;17(4):1191.

8. Nie W, Xu P, Hao C, Chen Y, Yin Y, Wang L. Efficacy and safety of over-the-counter analgesics for primary dysmenorrhea: A network meta-analysis. Medicine (Baltimore). 2020;99(19):e19881.

9. Uysal G, Akkaya H, Cagli F, Tutus S, Tayyar AT. A comparison of two different oral contraceptives in patients with severe primary dysmenorrhoea. J OBSTET GYNAECOL. 2018;38(6):828-32.

10. Szamosi S. [Recommendation for the substantial choice between various nonsteroidal antiinflammatory drugs based on their safety profile]. Orv Hetil. 2018;159(44):1783-8.

11. Dragoman MV, Tepper NK, Fu R, Curtis KM, Chou R, Gaffield ME. A systematic review and metaanalysis of venous thrombosis risk among users of combined oral contraception. Int J Gynaecol Obstet. 2018;141(3):287-94.

12. Leem J, Jo J, Kwon CY, Lee H, Park KS, Lee JM. Herbal medicine (Hyeolbuchukeo-tang or Xuefu Zhuyu decoction) for treating primary dysmenorrhea: A systematic review and meta-analysis of randomized controlled trials. Medicine (Baltimore). 2019;98(5):e14170.

13. Gao Y, Wang GQ, Xie YM, Wei RL, Liu FM. [Study of integrative evidence chain in clinical trial of Guizhi Fuling Capsules based on "Yibing Tongzhi" in clinical applications]. Zhongguo Zhong Yao Za Zhi. 2020;45(10):2304-9.

14. Peng $Y$, Zheng X, Fan Z, Zhou H, Zhu X, Wang G, et al. Paeonol alleviates primary dysmenorrhea in mice via activating CB2R in the uterus. PHYTOMEDICINE. 2020;68:153151.

15. Cheng Y, Chu Y, Su X, Zhang K, Zhang Y, Wang Z, et al. Pharmacokinetic-pharmacodynamic modeling to study the anti-dysmenorrhea effect of Guizhi Fuling capsule on primary dysmenorrhea rats. PHYTOMEDICINE. 2018;48:141-51.

16. Sun L, Liu L, Zong S, Wang Z, Zhou J, Xu Z, et al. Traditional Chinese medicine Guizhi Fuling capsule used for therapy of dysmenorrhea via attenuating uterus contraction. J ETHNOPHARMACOL. 
2016;191:273-9.

17. Xiong Z, Lang L, Gao X, Xiao W, Wang Z, Zhao L. An integrative urinary metabolomic study of the therapeutic effect of Guizhi Fuling capsule on primary dysmenorrheal rats based (1)H NMR and UPLC-MS. J Pharm Biomed Anal. 2019;164:750-8.

18. Wang GQ, Gao Y, Liu FM, Wei RL, Xie YM. [Post-marketing surveillance on Guizhi Fuling Jiaonang based on literature review]. Zhongguo Zhong Yao Za Zhi. 2018;43(4):820-32.

19. Schnyer RN, McKnight P, Conboy LA, Jacobson E, Ledegza AT, Quilty MT, et al. Can Reliability of the Chinese Medicine Diagnostic Process Be Improved? Results of a Prospective Randomized Controlled Trial. J Altern Complement Med. 2019;25(11):1103-8.

20. Wu T, Bian Z, Shang H, Li Y. Innovation of clinical trials in China: Commentary on the publication of “CONSORT extension for Chinese herbal medicine formulas 2017: Recommendations, explanation, and elaboration". Journal of Evidence-Based Medicine. 2017;10(3):155-62.

21. Chan AW. SPIRIT 2013 Statement: defining standard protocol items for clinical trials. ANN INTERN MED. 2013;158(3):200-7.

22. Zhao XM, Zhang Y, He XH, Chen HD, Wang ZF, Guo J, et al. Chinese herbal medicine Shenzhuo Formula treatment in patients with macroalbuminuria secondary to diabetic kidney disease: study protocol for a randomized controlled trial. 2018;19(1):200.

23. Lyu YR, Yang WK, Park SJ, Kim SH, Kang WC, Jung IC, et al. Efficacy and safety of GHX02 in the treatment of acute bronchitis: protocol of a phase II, double-blind, randomised placebo-controlled trial. BMJ OPEN. 2018;8(5):e19897.

24. Sima Younesy SASE. Effects of Fenugreek Seed on the Severity and Systemic Symptoms of Dysmenorrhea. J Reprod Infertil. 2014:41-8.

25. Yang J, Chen J, Lao L, Yang M, Chen J, Bo L, et al. Effectiveness study of moxibustion on pain relief in primary dysmenorrhea: study protocol of a randomized controlled trial. 2014;2014:434978.

26. Tang L, Jia P, Zhao L, Kang D, Luo Y, Liu J, et al. Acupuncture treatment for knee osteoarthritis with sensitive points: protocol for a multicentre randomised controlled trial. BMJ OPEN. 2018;8(10):e23838.

27. Aghamohammadi D, Ayromlou H, Dolatkhah N, Jahanjoo F, Shakouri SK. The effects of probiotic Saccharomyces boulardii on the mental health, quality of life, fatigue, pain, and indices of inflammation and oxidative stress in patients with multiple sclerosis: study protocol for a doubleblind randomized controlled clinical trial. 2019;20(1):379.

28. Lee H, Choi TY, Myung CS, Lee MS. Herbal medicine Shaofu Zhuyu decoction for primary dysmenorrhea: a systematic review protocol. 2016;5:9.

29. Kashefi F, Khajehei M, Tabatabaeichehr M, Alavinia M, Asili J. Comparison of the effect of ginger and zinc sulfate on primary dysmenorrhea: a placebo-controlled randomized trial. 2014;15(4):826-33.

30. Park J, Park S, Cheon C, Go H, Sun S, Shin Y, et al. Effects of Gyejibongnyeong-hwan on dysmenorrhea caused by blood stagnation: study protocol for a randomized controlled trial. TRIALS. 2012;13(1):3. 
31. Jiayang He ZLWH. Efficacy and safety of Chou-Ling-Dan granules in the treatment of seasonal influenza via combining Western and traditional Chinese medicine: protocol for a multicentre, randomised controlled clinical trial. BMJ OPEN. 2018;9(4):e24800.

32. Agarwal G, Pirrie M, McLeod B, Angeles R, Tavares W, Marzanek F, et al. Rationale and methods of an Evaluation of the Effectiveness of the Community Paramedicine at Home (CP@home) program for frequent users of emergency medical services in multiple Ontario regions: a study protocol for a randomized controlled trial. 2019;20(1):75.

33. Abdulahi M, Fretheim A, Magnus JH. Effect of breastfeeding education and support intervention (BFESI) versus routine care on timely initiation and exclusive breastfeeding in Southwest Ethiopia: study protocol for a cluster randomized controlled trial. 2018;18(1):313.

34. Abaraogu UO, Dall PM, Brittenden J, Stuart W, Tew GA, Godwin J, et al. Efficacy and Feasibility of Pain management and Patient Education for Physical Activity in Intermittent claudication (PrEPAID): protocol for a randomised controlled trial. 2019;20(1):222.

35. Songqiao Liu CYJZ. Efficacy of Xuebijing Injection for Sepsis (EXIT-SEP): protocol for a randomised controlled trial. 2018;9(8):e28664.

36. Yu S, Wen Y, Xia W, Yang M, Lv Z, Li X, et al. Acupoint herbal plaster for patients with primary dysmenorrhea: study protocol for a randomized controlled trial. 2018;19(1):348.

37. Agnelli G, Becattini C, Bauersachs R, Brenner B, Campanini M, Cohen A, et al. Apixaban versus Dalteparin for the Treatment of Acute Venous Thromboembolism in Patients with Cancer: The Caravaggio Study. 2018;118(9):1668-78.

38. Abdollahi S, Salehi-Abargouei A, Tabatabaie M, Sheikhha MH, Fallahzadeh H, Rahmanian M, et al. The effect of resveratrol supplementation on the expression levels of factors associated with cellular senescence and SCD163/sTWEAK ratio in patients with type 2 diabetes mellitus: study protocol for a double-blind controlled randomised clinical trial. 2019;9(7):e26337.

39. Ana E S Jorge LODP. Photobiomodulation therapy associated with supervised therapeutic exercises for people with knee osteoarthritis: a randomised controlled trial protocol. BMJ OPEN. 2019;10(6):e35711.

40. He H, Chen G, Gao J, Liu Y, Zhang C, Liu C, et al. Xue-Fu-Zhu-Yu capsule in the treatment of qi stagnation and blood stasis syndrome: a study protocol for a randomised controlled pilot and feasibility trial. 2018;19(1):515.

\section{Figures}




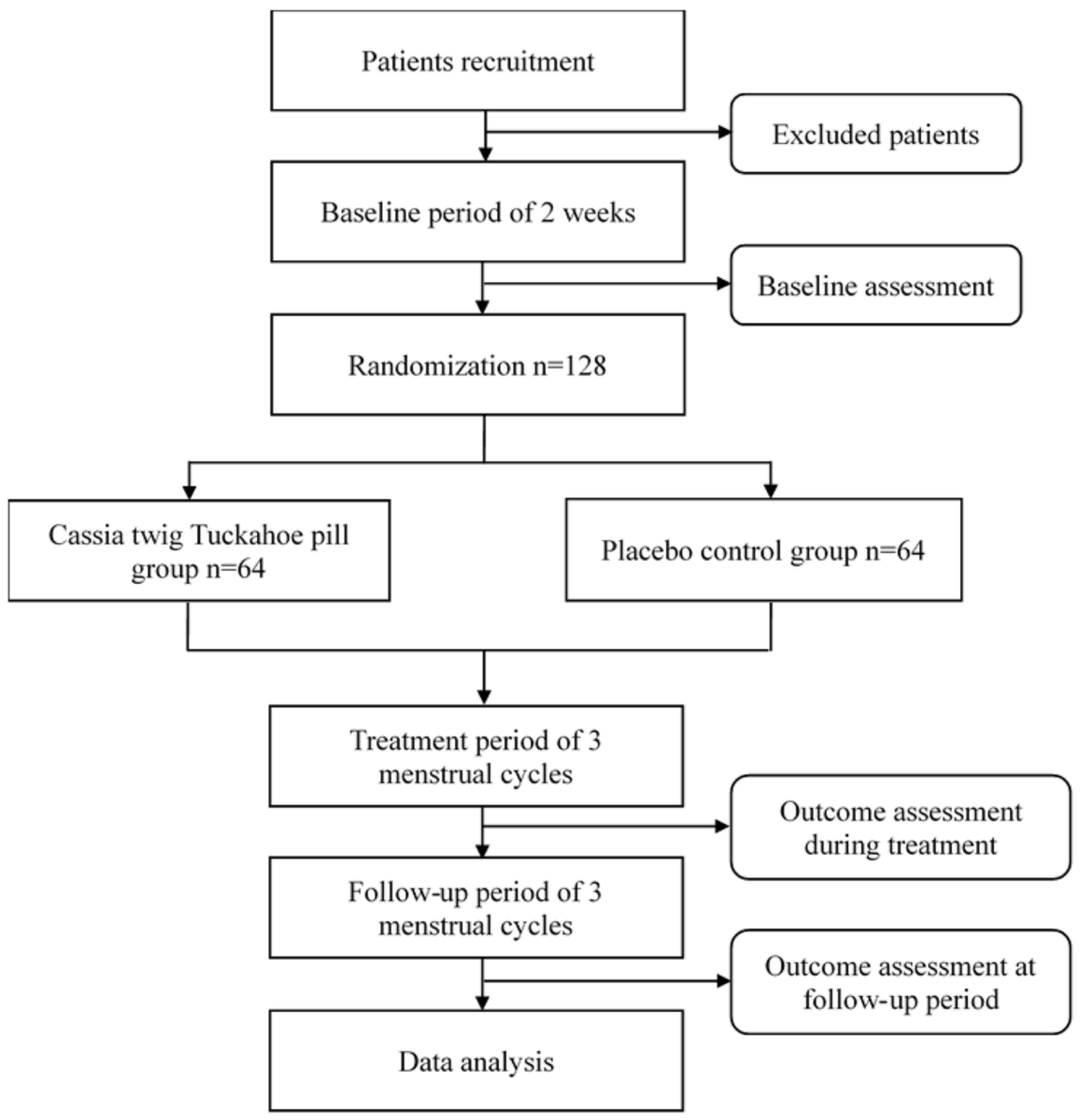

Figure 1

Retention and reasons for the withdrawal of participants throughout the trial will be documented [34]. The outcome measures will be described according to Figure1.

\section{Supplementary Files}


This is a list of supplementary files associated with this preprint. Click to download.

- SPIRITchecklist1.docx 